\title{
EDITORIAL
}

\section{Reporting guidelines for implementation and operational research}

\author{
Dermot Maher
}

http://dx.doi.org/10.5588/pha.16.0018

$\mathrm{n}$ public health, implementation research aims to improve understanding as to how to facilitate access to interventions that have been shown to work but have not reached many of the people who could benefit from them. Researchers identify practical problems facing health programmes, and aim to evaluate proposed solutions that can improve health outcomes. In operational research, routinely collected programme data are used to uncover ways of delivering more effective, efficient and equitable health care.

Implementation and operational research are often considered together, as they share similar aims as practical forms of downstream health research and are both usually carried out in close collaboration between researchers and those involved in health programmes. Implementation and operational research take the output of basic and clinical research to the next stage in answering questions on how best to make the products of basic and clinical research-new drugs, diagnostics, vaccines and other health technologies-as widely, efficiently and equitably available as possible.

Guidelines have been developed for many different types of research aimed at enhancing research quality and transparency. ${ }^{1}$ As the fields of implementation and operational research utilise a range of different research designs, the existing research reporting guidelines only partially cover the need for guidance on implementation and operational research. Filling this gap, new reporting guidelines have been published for the growing area of implementation and operational research. ${ }^{2}$ The aim is to help to improve the quality of reports on these types of research, and in due course the quality of the research itself.

The guidelines are the outcome of wide consultation through the World Health Organization (WHO), the Special Programme for Research and Training in Tropical Diseases (TDR) and the Alliance for Health Policy \& Systems Research (AHPSR). The intention is to provide a practical reference for funders, researchers, policy makers, implementers, reviewers and editors involved in implementation and operational research. Given that this is an evolving field, the plan is to monitor the use of these guidelines and update them as required.

As the main scope of Public Health Action (PHA) covers implementation and operational research, the new guidelines are of particular value to the researchers in these fields who submit papers to this journal. The criteria for considering papers submitted to PHA for publication will include the extent to which they reflect the guidelines. In planning, carrying out and reporting on implementation and operational research, researchers should find the guidelines of practical value in improving research quality and increasing their chance of publication of their papers in this and other journals.

\section{References}

1 Enhancing the quality and transparency of health research (EQUATOR). Oxford, UK: Equator Network, 2015. http://www. equator-network.org/ Accessed January 2016

2 Hales S, Lesher-Trevino A, Ford N, Maher D, Ramsay A, Tran N. Reporting guidelines for implementation and operational research. Bull World Health Organ 2016; 94: 58-64. http:// www.who.int/bulletin/volumes/94/1/15-167585.pdf Accessed January 2016
AFFILIATIONS Tropical Diseases Research, World Health Organization, Geneva, Switzerland

\section{CORRESPONDENCE} Dermot Maher

Tropical Diseases Research World Health Organization Geneva, Switzerland e-mail: maherd@who.int

Conflicts of interest: none declared.

The author is a staff member of the World Health Organization (WHO). The author alone is responsible for the views expressed in this article and they do not necessarily represent the decisions, policy or views of the WHO.
Public Health Action (PHA) The voice for operational research. Published by The Union (www.theunion.org), PHA provides a platform to fulfil its mission, 'Health solutions for the poor'. PHA publishes high-quality scientific research that provides new knowledge to improve the accessibility, equity, quality and efficiency of health systems and services.
e-ISSN 2220-8372

Editor-in-Chief: Dermot Maher, MD, Switzerland

Contact: pha@theunion.org

PHA website: http://www.theunion.org/what-we-do/journals/pha Article submission: http://mc.manuscriptcentral.com/pha 